\title{
LA ORGANIZACIÓN Y LA MOVILIZACIÓN DE LOS PEQUEÑOS AGRICULTORES DEL ALTO URUGUAY FRENTE A LA CONSTRUCCIÓN DE LA REPRESA DE ITÁ, BRASIL (1979-1999)
}

\author{
THE ORGANIZATION AND MOBILIZATION OF SMALL FARMERS IN UPPER URUGUAY IN FRONT OF \\ THE CONSTRUCTION OF THE ITÁ DAM, BRAZIL (1979-1999)
}

\author{
María Rosa Catullo1 \\ ORCID: https://orcid.org/0000-0002-1060-1139 \\ Maria José Reis2 \\ ORCID: https://orcid.org/0000-0001-5394-0803
}

Submissão: 16/11/2020 / Aceito: 13/03/2021 / Publicado: 13/04/2021

\section{Resumen}

El trabajo tiene por objetivo analizar la movilización y las estrategias de los pequeños productores rurales afectados por la construcción de la represa de Itá, ubicada sobre el río Uruguay, entre los municipios de Itá (Estado de Santa Catarina) y Aratiba (Estado de Río Grande del Sur), con el fin de resistir y luchar por indemnizaciones y relocalizaciones para todos los afectados. Metodológicamente, la investigación se caracteriza como cualitativa. Comprende revisión de la literatura, lectura crítica de documentación de la Comissão Regional de Atingidos por Barragem (CRAB) y entrevistas con agricultores afectados y líderes de la CRAB. Hemos constatado que los agricultores del municipio de Itá se organizaron y formaron la Comissão Regional de Atingidos por Barragem (CRAB) desde sus inicios (1979) y lograron a fines de 1987 firmar, por primera vez en la historia de las disputas de los afectados por represas y las empresas del sector eléctrico brasileño, un Acuerdo con ELETROSUL por el cual se indemnizaba y relocalizaba a todos los afectados rurales de dicha represa, propietarios y no propietarios.

Palabras claves: Conflictos socio ambientales. Relocalización compulsiva de población. Represa de Itá. Pequeños agricultores. CRAB.

Abstract
In this work aims to analyze the mobilization and strategies of small rural producers affected by the construction of the Itá dam, located on the Uruguay River, between the municipalities of Itá (State of Santa Catarina) and Aratiba (State of Rio Grande del Sur), in order to resist and fight for compensation and relocations for all those affected. Methodologically, research is characterized as qualitative. It includes literature review, critical reading of documentation from the Regional Commission of Atingidos by Barragem (CRAB) and interviews with affected farmers and CRAB leaders. We have found that farmers in the municipality of Itá have organized and formed the

\footnotetext{
${ }^{1}$ Dra. en Antropología. Instituto de Relaciones Internacionales de la Universidad Nacional de La Plata. Investigadora del Consejo Nacional de Investigaciones Científicas y Técnicas. E-mail. mcatullo2000@yahoo.com.ar. La Plata, Argentina.

${ }^{2}$ Dra. en Antropología y Prof. e Investigadora Jubilada. Trabajó en el Departamento de Antropología de la Universidad Federal de Santa Catarina, Programa de Posgrado, y en la Universidade do Vale de Itajai. E-mail: masereis43@gmail.com. Florianópolis, Brasil.
}

DOI: https://doi.org/10.46699/rduno.v3i4.5879 | Edição Vol. 3, Núm. 4, 2020. 
Regional Commission of Atingidos by Barragem (CRAB) since its inception (1979) and managed at the end of 1987 to sign, for the first time in the history of disputes over dams and companies in the Brazilian electricity sector, an agreement with ELETROSUL in which all rural affected persons of that dam were compensated and relocated, owners and non-owners.

Keywords: Socio-environmetal conflicts. Compulsilve Population Relocation. Itá Dam. Small farmers. CRAB.

\section{INTRODUCCIÓN}

La producción de energía en Brasil, a partir de las primeras décadas del siglo pasado, se logró, sobretodo, mediante el uso de recursos hídricos.

El proceso de la construcción de las represas hidroeléctricas, en especial las de gran porte, implica la participación de una serie de agencias y actores sociales. Entre ellos se destacan las agencias nacionales de regulación del sector eléctrico, los consorcios públicos o privados responsables por la ejecución de proyectos hidroeléctricos y las poblaciones regionales y locales, que ocupan las áreas requeridas para su instalación.

Las investigaciones nacionales e internacionales han demostrado que en el ámbito regional y local la construcción de represas invariablemente implica un reordenamiento territorial y consecuencias sociales y ambientales importantes. Delante de la formación de un lago artificial, son inundadas vastas extensiones de tierra. También es necesario transformar y ocupar espacios para la construcción e instalación de otras obras de infraestructura, como caminos, puentes, líneas de transmisión, y el establecimiento de villas residenciales para técnicos y obreros responsables por la construcción y mantenimiento de las represas.

Una vez que comúnmente los espacios necesarios para la construcción y el funcionamiento de este tipo de obras no son "espacios vacíos", sino que alojan diferentes segmentos sociales - en Brasil más frecuentemente poblaciones indígenas y pequeños productores rurales que ocupan las tierras como su principal medio de producción- se torna necesario la retirada forzosa de estas poblaciones que históricamente venían ocupando estos espacios.

Por otro lado, es indispensable destacar que no solamente las poblaciones removidas de las áreas requeridas para la instalación de centrales hidroeléctricas han sufrido los efectos sociales y ambientales derivados de ellas. En rigor, la ejecución de tales obras, además de sus beneficiarios entre los que se encuentran grupos empresariales e industriales, contratistas y consorcios de emprendedores- termina creando miles de víctimas. Las denominadas por Renk y Winckler (2017) 
como "Victimas del desarrollo", entre ellas, las poblaciones que permanecen en los espacios alterados. También se ve mutilado su patrimonio natural y su espacio social3.

En el presente artículo analizamos los efectos de la construcción de la represa de Itá en los pequeños agricultores del municipio homónimo. La presa está ubicada en el Alto Uruguay, próxima a la desembocadura del río Uvá, entre los municipios de Itá (Estado de Santa Catarina) y Aratiba (Estado de Río Grande del Sur). La misma formó un lago con una cota de 370 metros, con un volumen total de 5.100 millones de metros cúbicos, ocupando $141 \mathrm{Km}^{2}$ de área total; e implicó la inundación de 2806 propiedades rurales y 342 urbanas. Presenta un potencial instalado de 1620 MW e inundó en su gran mayoría áreas rurales localizadas en cinco municipios catarinenses (Itá, Concórdia, Ipira, Peritiba y Piratuba) y cuatro de Rio Grande del Sur (Aratiba, Marcelino Ramos, Mariano Moro y Severiano de Almeida) (ELETROSUL/CNEC, 1990, p. 90); y la ciudad de Itá.

De acuerdo a los datos oficiales en relación al número de personas que fueron reasentadas como consecuencia de la construcción de dicha represa, Eletrosul estimaba en 1997 el desplazamiento de cerca de 16.000 personas, entre las cuales más del 80\% eran pequeños productores rurales o campesinos. Es por esta razón que nos centramos en las estrategias y luchas de la población rural del municipio de Itá, ubicado en el valle del Alto Uruguay, estado de Santa Catarina.

Para tales fines nos hemos basado en los trabajos de campo de Reis, quien defendiera su tesis doctoral en 1998, en el Programa de Post-grado en Ciencias Sociales de la Universidad Estadual de Campinas. Además, hemos analizado críticamente la documentación de la CRAB (1979-1998); el periódico “A Enchente do Uruguai” que comenzó a salir en 1981, y que jugó un papel importante en la difusión de las posiciones del movimiento y en la consolidación de los fundamentos ideológicos para las movilizaciones; y los "Dossié Barragen" donde la CRAB recolectaba los artículos de diarios locales, regionales, nacionales e internacionales principalmente sobre la construcción de represas en todo Brasil, el sistema hidroeléctrico, la situación a mbiental entre otros temas. Los mismos fueron recolectados en trabajos de campo realizados por Catullo y Reis en los años 1999, 2001 y 2003 en la ciudad de Itá y de Erexim (RS), sede de la CRAB. Asimismo, inspeccionamos la documentación de Centrais Elétricas do Sul do Brasil (Eletrosul) y de Centrais

\footnotetext{
${ }^{3}$ Respecto a las pérdidas en relación al patrimonio cultural y natural, consecuencia de la instalación de represas hidroeléctricas, véase, entre otros, Reis (1998) y Reis; Catullo e Castells (2003).
} 
Geradoras do Sul do Brasil S.A. (Gerasul) y realizamos entrevistas a funcionarios y técnicos en la sede de Gerasul, en la ciudad de Florianópolis.

\section{HISTORIA DEL GRAN PROYECTO}

Históricamente, la implementación de las UHEs en la Cuenca del Río Uruguay fue el resultado de estudios realizados para la planificación y desarrollo del sector energético en los años 70 por el Consorcio Canadiense Americano-brasileño - Canambra (Winckler y Renk, 2019, p. 85).

Eletrosul, con miras a reemplazar las centrales termoeléctricas por centrales hidroeléctricas, aprovechando el potencial hidráulico de la cuenca para abastecer de energía al país, presentó en noviembre de 1979 el Inventario Hidroeléctrico de la Cuenca del Río Uruguay, o el llamado "Proyecto Río Uruguay", que preveía la instalación de 22 represas (19 centrales hidroeléctricas y 3 para regular los caudales de agua), con 7 centrales prioritarias, a saber: Machadinho, Itá, Campos Novos, Barra Grande, Iraí e Itapiranga (Winckler y Renk, 2019, p. 85). Entre 1980 y 1985 se resolvió que la primera represa a construir sería la represa de Itá, luego la de Machadino, tercera la de Campos Novos, y la última sería la de Barra Grande.

En el año 1988 se modificó la Legislación Ambiental brasileña y se realizó el "Relatorio de Impacto Ambiental” (RIMA) de la represa de Itá.

La construcción de la represa comenzó a inicios de los 80 y fue paralizada en el año 1985, a raíz del movimiento formado a partir de 1979, y contrario a la construcción de represas: la Comissão Regional de Atingidos por Barragem (CRAB). Después de muchos enfrentamientos y conflictos, y de la constante presión de la CRAB y la población campesina, la represa se reinició con posterioridad al Acuerdo firmado entre Eletrosul y la CRAB en el mes de octubre de 1987, en el cual el ente energético del sur de Brasil se comprometía a indemnizar y relocalizar a los "colonos" afectados.

Eletrosul fue privatizada por el Poder Ejecutivo en diciembre de 1997. En consecuencia, se dividió en dos partes. Por un lado, Gerasul, que quedó con la construcción de represas, y por el otro Eletrosul, que se hizo cargo de la distribución, de subestaciones y de las líneas de trasmisión. Posteriormente, en subasta realizada el 15 de setiembre de 1998, Tractebel Sul Ltda., actualmente denominada Tractebel EGI South America Ltda., empresa constituida en Brasil bajo el control de Tractebel Sociètè Anonyme, con sede en Bruselas, Bélgica, adquirió el control accionario de Gerasul. Ya en julio de 2016 Tractebel Energia pasó a formar parte de ENGIE Brasil Energia. 
La represa de Itá se inauguró el 16 de diciembre de 1999 y el lago comenzó a llenarse inundando completamente la antigua ciudad homónima y grandes áreas rurales.

\section{LA MOVILIZACIÓN POLÍTICA DE LOS PEQUEÑOS AGRICULTORES DEL ALTO URUGUAY ORGANIZADOS EN LA CRAB}

Varios autores han señalado que la tradición de Eletrobrás (Centrais Elétricas Brasileiras S. A.) y de sus distribuidoras, ha sido ignorar las implicaciones sociales de sus iniciativas en el momento de la toma de decisiones. En este sentido, Sigaud refiere que "Una vez definido qué se va a realizar el aprovechamiento de una cuenca o de un río y habiendo ya firmados los contratos millonarios con las empresas e incluso adquirido equipos pesados y caros, es recién entonces que se procede a lo que se ha convenido llamar de 'evaluación de impactos sociales '” (1988, p.108, nuestra traducción).

Esta apreciación, “...tendría como finalidad apenas 'minimizar', 'mitigar', o 'neutralizar' impactos de una decisión ya tomada o en curso, y nunca una evaluación de 'impactos' para ser tenida en cuenta en la decisión de construir una represa hidroeléctrica", lo que ha sido priorizado por el sector eléctrico en la toma de decisión, es la producción de energía, ocurriendo negligencia e irresponsabilidad en relación a las implicaciones sociales de este tipo de inversión. Así sucedió con la ejecución de las represas de Itaipú (Germani, 2003), Sobradinho (Sigaud, 1986; Martins Costa, 1990), Itaparica (Pandolfi, 1986) y Tucuruí (Magalhães, 1996).

En la práctica, en relación a las providencias relativas a la expropiación y desplazamiento de las poblaciones que ocupan las áreas destinadas a la implementación de las represas hidroeléctricas, las subsidiarias de Eletrobras han actuado de forma semejante, de acuerdo con tres estrategias básicas (Vainer y Araújo, 1990, 20): la desinformación; la perspectiva territorial patrimonialista y la negociación individual. A estas estrategias, consideramos indispensable sumar como marca de esta actuación una postura autoritaria y prepotente en el trato con las poblaciones locales.

En este sentido, es necesario recordar que la igualdad entre las partes que se supone presente en un contrato particular de compra y venta no pasa, en estas condiciones de una ficción legal: de un lado está una gran empresa estatal, con sus enormes recursos, sus cuerpos técnicos y jurídicos, es decir, el poder; del otro lado está el pequeño productor, desamparado, temeroso, presionado (Vainer y Araújo, 1990, p.21). En esta relación asimétrica, están enfrentadas, dos lógicas diferentes y contradictorias. De un lado la empresa a quien le interesa el emprendimiento, cuyos objetivos son legitimados por la ideología del desarrollo y del progreso. Del otro, las poblaciones locales para los 
que está en juego la expropiación de sus tierras, su permanencia en el área y la posibilidad de mantener su forma de vida.

Eletrosul trató, en el caso de la represa de Itá, de reproducir, en parte, estas mismas prácticas tradicionales. Asumió, desde el inicio, la perspectiva tradicionalista; insistió en las negociaciones individuales, en el caso de las indemnizaciones; ocultó información sobre el Proyecto; minimizó sus aspectos negativos y pregonó ventajas; esquivó, hasta donde fue posible, el diálogo con los pequeños productores, además de, autoritariamente, invadir propiedades rurales. Pero, la reacción de las poblaciones locales, de enfrentamiento con Eletrosul, especialmente en el caso de las represas de Itá y Machadinho, se anticipó al inició de la implantación de las obras, lo que acabó por interferir en el modo tradicional de la empresa (Reis, 1998, p. 48)

Esta reacción se tornó posible gracias, por lo menos, a tres factores de acuerdo a SchererWarren y Reis (1986). En primer lugar, la atenuación de la represión política ejecutada por los gobiernos militares y el inicio del proceso de democratización, para lo cual, sin duda, contribuyeran mucho estos movimientos. Y en este sentido, la Ley de Amnistía, de agosto de 1979, permitió la vuelta de los exiliados políticos al país, reforzando el activismo en diversas causas sociales (Rocha y Lima Oliveira, 2018, p.35-36). En segundo lugar, los cambios estructurales en la economía agraria con el incremento del proceso de modernización agrícola y las consecuentes alteraciones en los patrones de uso de la tierra, en la tecnología y en las relaciones de trabajo en el campo, bien como el acceso a políticas estatales. Por último, la presencia y actuación en la arena política de sectores de la Iglesia Católica y en menor proporción, de la Iglesia de Confesión Luterana en Brasil, y de los sindicatos de trabajadores rurales, desempeñándose como mediadores, esto es, individuos y grupos que brindaron apoyo material y simbólico para la incipiente movilización de los afectados por las represas. (Idem, p. 36).

Así, en el caso específico del Alto Uruguay, tanto de Rio Grande del Sur, como de Santa Catarina, al entrar en escena la problemática de la instalación de represas, los pequeños productores rurales ya contaban con la presencia de esos "mediadores" involucrados en la movilización popular; en luchas por la conquista de tierras de aquellos que habían sido expropiados de las mismas; por el precio de los productos; por el establecimiento de una adecuada política agrícola, entre otras cuestiones (Reis, 1998, p. 48-49)

Eletrosul, a diferencia de los procesos de implantación de represas hidroeléctricas en otras regiones brasileñas, tuvo que enfrentar, desde el inicio, una fuerte reacción de las poblaciones rurales locales, constituidas, en su mayoría de pequeños productores rurales. 
Después de que fueran publicados los resultados de la revisión de los estudios específicos del aprovechamiento hidroenérgetico del río Uruguay y sus afluentes (octubre de 1979), y el estudio sobre la viabilidad de las represas de Itá y Machadinho (noviembre del mismo año), sucedió, por iniciativa de la "Comissão Pastoral da Terra" (en adelante, CPT), una primera reunión para discutir la problemática en cuestión. Realizada en la ciudad de Chapecó el 27 y 28 de diciembre de 1979, contó con la presencia de pequeños productores de los estados de Paraná, Santa Catarina y Río Grande del Sur, representantes de la CPT de Santa Catarina y Rio Grande del Sur, agentes pastorales, vicarios de las Iglesias Católicas y Pastores de la Iglesia Evangélica de Confesión Luterana, sociólogos y agrónomos de la "Fundação do Alto-Uruguai para a Pesquisa e Ensino Superior (FAPES)". En esta primera reunión se tomó la decisión de crear una "Comissão de Barragens", destinada a pensar y enfrentar los problemas más importantes.

A partir de esta iniciativa, Eletrosul tuvo que modificar el rumbo de las decisiones sobre las "cuestiones sociales", especialmente en relación al desplazamiento de los productores rurales.

Posteriormente, en una reunión realizada en la ciudad de Concórdia en marzo de 1980, la "Comissão de Barragens", con vistas a la propuesta de la creación de "comisiones locales y municipales", recibió la denominación de "Comissão Regional de Atingidos por Barragens" (CRAB) (Reis, 1998, p.52). En poco tiempo, las comisiones se multiplicaron, principalmente, las locales, constituidas a menudo por las propias "Comunidades Eclesiais de Base" (CEBs) o, de una forma u otra, transmitidas a las comunidades tradicionales.

Sin duda alguna, la configuración y la dinámica de la reacción de los pequeños productores al "Proyecto Uruguay" tuvo que ver directamente con la actuación de esta Comisión. Es la que divulgó la noticia de la construcción de las represas hidroeléctricas de Itá y Machadinho, y contrapuso al discurso de "progreso" y de beneficios para la región que planteaba Eletrosul, una identificación de "daños y pérdidas" para los pequeños productores ocupantes de las áreas requeridas por estos grandes emprendimientos.

En términos operacionales, la CRAB estaba constituida, hasta fines de 1983, por una Secretaría, localizada en Erexim (RS), "responsable por los trabajos burocráticos e por la coordinación general del movimiento" (CRAB, 1985, nuestra traducción). En inicio de 1984, líderes de la CRAB decidieron la creación de una "Ejecutiva", compuesta por representantes de afectados de varias regiones de la Cuenca del Río Uruguay, y comenzaron movilizaciones junto a sindicalistas rurales y personal de la Secretaria. En 1986, además de las Comisiones Regionales creadas en 1985, de la Secretaria y de la Ejecutiva, instituyeron la Asamblea como "un espacio de discusión y 
deliberación del Movimiento, de inicio anual y posteriormente bianual" (CRAB,1990) (SchererWarren e Reis, 1989, p. 52-53)

De acuerdo a Reis, es a través de esta identificación que las "victimas" pasaron a ser reconocidas y políticamente auto-denominadas de "atingidos" (afectados), constituyéndose en nuevos sujetos sociales. A través de la actuación de la CRAB se pudo buscar y transmitir información sobre las consecuencias y magnitud del Gran Proyecto; encaminar demandas y reivindicaciones; definir enemigos y aliados; presionar a intendentes y concejales para que se posicionaran; golpear las puertas de las Asambleas Legislativas tanto de Rio Grande del Sur como de Santa Catarina; recurrir a Brasilia cuando fuera necesario y presentar una suscripción de más de un millón y medio de firmas. (1998, p. 53). Es también a través de su actuación que se constituyó y adquirió visibilidad el "Movimento dos Atingidos por Barragens" (MAB), organizando grandes festivales, publicando un periódico, armando programas de radio, creando "hechos políticos", tal como la retención de técnicos de Eletrosul o la invasión de campamentos en los "territorios" ocupados por dicha empresa. Asimismo, fue con la actuación de la CRAB que se establecieron los términos del "Acuerdo" firmado entre Eletrosul y la CRAB en octubre de 1987, en relación a soluciones para la retirada de los "atingidos", incluyendo entre estas el reasentamiento de los afectados no propietarios de tierras.

Reconocer, el papel fundamental de la $\mathrm{CRAB}$ en la organización y movilización de los “atingidos" de la Cuenca del rio Uruguay, no significa ignorar que su actuación no puede ser vista simplemente como producto de sus propias iniciativas y de la voluntad y creatividad de sus dirigentes.

Es imposible desconocer la presencia y la influencia de los diferentes mediadores ya mencionados, especialmente de la llamada "Iglesia Popular"4 y de los Sindicatos de Trabajadores Rurales, cuyas marcas son imperceptibles a lo largo de toda la trayectoria del Movimiento y de la actuación de la CRAB.

\section{FE Y VIDA. LA INTERMEDIACIÓN DE LA "IGLESIA PROGRESISTA"}

La movilización de los pequeños productores rurales en relación al problema de represas en el rio Uruguay contó, además de la implicación de varios tipos de agentes, de la Iglesia Católica y

\footnotetext{
${ }^{4}$ Fracción de la Iglesia Católica cuya actuación fue orientada por la "Teología de la Liberación”, tomando como marcos al Concilio Vaticano II (final de los años 50) y, en América Latina, las reuniones del episcopado realizadas en la ciudad de Puebla y Medellín (1968 y 1979), respectivamente.
}

DOI: https://doi.org/10.46699/rduno.v3i4.5879 | Edição Vol. 3, Núm. 4, 2020. 
pastores de la Iglesia Protestante de Confesión Luterana, con la presencia y la adhesión de su propia jerarquía representada por obispos de varias diócesis del norte de Rio Grande del Sur y del Oeste catarinense.

Aparte de la participación directa o indirecta de su jerarquía y la actuación políticamente comprometida de los sacerdotes, pastores y religiosas, anunciando y alertando en los púlpitos y otros espacios religiosos como las "Comunidades Eclesiales de Base"(CEBs) sobre la construcción de represas hidroeléctricas en la Cuenca del rio Uruguay, la presencia de la Iglesia fue decisiva, también en el sentido de movilizar y organizar a los pequeños productores rurales para el problema en cuestión, de diversas otras maneras. Decisiva aportando su infraestructura física para la realización de eventos, tales como cursos, asambleas, etc. Decisiva, intermediando la obtención de recursos financieros en la propia región y hasta internacionalmente. 5 Pero sobre todo, indispensable por su actuación político-pedagógica orientada a la formación de líderes que fueran, en el inicio de la reacción contra las represas, siendo los principales agentes movilizadores.

Como afirma Navarro (apud Moraes, 1994, p.130) por lo menos 90\% de los cuadros dirigentes e intermediarios de todos los movimientos sociales, en el período (1979 a 1986) "empezaron a comprender de la mano de la Iglesia". La formación de esos cuadros fue llevada a cabo por la "Escola Diocesana de Servidores de Erexim" (ESC), por la Pastoral da Juventude (PJ), y por la Comissão Pastoral da Terra (CPT),

Como en otros movimientos populares, donde estuvo presente el trabajo movilizador de estos líderes preparados por la "Iglesia Progresista", sean rurales o urbanos, signos de lenguaje como "La caminada", el "pueblo oprimido" y la "liberación" fueron presencias constantes en el discurso sobre la problemática que analizamos, especialmente, a través del habla de sus agentes en grandes manifestaciones públicas. Proliferaban, también, en esos discursos, imágenes bíblicas que metafóricamente fueran apropiadas a través del ejercicio realizado en las CEB, de aproximación entre "Fe y vida" para pensar la cuestión de las represas. De esta manera, la formación de los lagos en función por su construcción más que una inundación sería un “diluvio". (CRAB, 1982, p.144).

\footnotetext{
5 Sobre este aspecto, existe una detallada información en el trabajo de Rothman (1993).
}

DOI: https://doi.org/10.46699/rduno.v3i4.5879 | Edição Vol. 3, Núm. 4, 2020. 


\section{ENTRE LA "LUCHA MAYOR" Y LAS "LUCHAS ESPECÍFICAS": LAS ESCUELAS DE LOS SINDICATOS Y LAS ASESORÍAS TÉCNICAS}

Si hasta mediados de la década del 80 las actividades de formación de líderes rurales en el Alto Uruguay estuvieron a cargo de órganos vinculados a la "Iglesia Progresista", a partir de ahí esta tarea es prioritariamente asumida por las Escuelas de los Sindicatos (Moraes, 1994, p. 161). Debido a las diversas articulaciones sindicales realizadas en los estados del sur y al crecimiento de sus actividades se fundó en 1985 la "Escuela Sindical Margarida Alves", (ESMA) por donde pasaron muchos de los dirigentes de los movimientos sociales del Alto Uruguay.

Esta nueva orientación en el modo de conducir las tareas de formación, alteró también, la relación con otros mediadores, las entidades de apoyo, como el "Centro de Educación Popular" (CEPO) fundado en 1986. Destinado a ejercer tareas tales como la formación de líderes, de preparación del material para comunicación - inclusive el boletín "A Enchente do Uruguai”- y de intermediación para la obtención de recursos financieros del exterior (Scherer Warren y Reis, 1989), luego se centró en la prestación de otros servicios y menos en tareas de formación.

Por otro lado, en el mismo año de la creación de los CEPO se instaló en el Alto Uruguay el Centro de Tecnología Alternativa (CETAP). El centro se creó con los objetivos de "relevar, investigar y difundir técnicas que se adapten a las pequeñas propiedades; prestar un servicio de asesoramiento en el área tecnológica a las organizaciones y movimientos ligados a la pequeña propiedad y entrenar y capacitar técnicos y productores" (CRAB, 1986, p.8, nuestra traducción)

El papel de las asesorías "técnicas", entre las cuales se incluía, también, la presencia de especialistas de diferentes áreas del conocimiento (agrónomos, sociólogos, geógrafos, abogados, etc.) fue fundamental para capacitar a la $\mathrm{CRAB}$ a tratar con un amplio espectro de demandas y cuestiones que la habían desafiado a transitar por el universo de la política energética, por los aspectos técnicos relativos a la construcción de represas hidroeléctricas y sus consecuencias socio ambientales, o para planificar e instalar proyectos de asentamientos rurales, además de las tareas esenciales de movilizar, concientizar y preparar a los pequeños productores rurales del Alto Uruguay para la lucha y reacción a la construcción de centrales hidroeléctricas. 


\section{DEMANDAS Y REIVINDICACIONES}

Mientras que los años de 1984 a 1986 estuvieron marcados por cambios en las perspectivas organizativas y políticas del Movimiento, en cuanto a sus reivindicaciones y demandas del Movimiento fue 1987 que trajo consigo la mayor novedad: el "Acuerdo" firmado entre la CRAB, reconocida a partir de entonces, como legitima representante de los "afectados", y ELETROSUL, siendo este último responsable, a través de él, de asumir ciertas "soluciones" en relación al desplazamiento de los pequeños productores rurales debido a la implementación de las centrales hidroeléctricas de Itá y Machadinho.

Este "Acuerdo", constituyó un marco que dividió la trayectoria del Movimiento en un “antes" y un "después" en relación a sus conquistas. Sin embargo, estas pueden dimensionarse adecuadamente en relación con el futuro del Movimiento y a la suerte de los afectados, ubicándolos en el propio pasado del Movimiento. Sin embargo, se dio durante el período de luchas que comenzaron ya en 1979, con las primeras reuniones para discutir las implicaciones, para las poblaciones locales, de la construcción de represas en la Cuenca del Uruguay.

Este período anterior a la firma del Acuerdo, en relación a las reivindicaciones del Movimiento se puede pensar que está compuesto por dos momentos: el primero, marcado por la búsqueda de soluciones, especialmente en relación al desplazamiento de los " afectados", que se inició en 1979 y se extendió hasta 1983; y el segundo -que comenzó en este último año y se prolongó hasta 1987, año de negociaciones y firma del Acuerdo- en el que en términos de retórica, se asume el rechazo total a la construcción de las represas. Es ésta la historia vivida por el Movimiento en los dos momentos, una historia de demandas, reivindicaciones, enfrentamientos y conquistas.

[...] sentimos que la organización es fuerte y aún mejor que eso: sentimos que estamos contribuyendo para que esta organización de los afectados se fortifique cada vez más, porque esa es la única forma de garantizarnos que nuestra tierra continúe siendo nuestra vida (agricultor afectado, Enchente do Uruguai, No 18, set. 1987, traducción nuestra).

[...] el momento es histórico. Tal vez nunca haya pasado en Brasil una reunión de negociaciones entre los afectados y la empresa constructora antes del inicio de la obra, y lo que es más importante: los afectados tienen protestas concretas para la solución de su problema, contempladas en el Documento, y exigen que se cumpla (Enchente do Uruguai, No 20, febrero, 1988, traducción nuestra). 


\section{GARANTIZAR EL ACUERDO}

[...] el cumplimiento de este acuerdo no depende de ELETROSUL. Sólo la lucha, la organización, la movilización de los afectados asegurará que se cumplan las palabras de este acuerdo. Todavía tenemos mucha lucha para garantizar nuestra tierra, que es nuestra vida. (CRAB, 1987, traducción nuestra).

Poco tiempo después, en abril de 1988, la CRAB denunciaba que ELETROSUL no cumplía adecuadamente el Acuerdo6 en la región de la represa de Itá.

Paralelamente, el Movimiento abrió nuevos frentes de lucha y asumió nuevas "banderas". Por un lado, fue uno de los principales organizadores del I Encuentro Nacional de Represas, llevado a cabo en Goiània en 1989, que dio origen a una nueva articulación nacional con otros Movimientos en torno al mismo problema. Por otro lado, se articuló a toda una red de Movimientos y Organizaciones No Gubernamentales (ONGs), que tenían las cuestiones ecológicas como centro de sus preocupaciones7, participando de eventos nacionales e internacionales.

\section{CONSIDERACIONES FINALES}

Podemos afirmar que los pequeños agricultores del Alto Uruguay formaron un movimiento popular: la CRAB, entendido, como explica Maria Stella Moraes, como "un colectivo de dirigentes y líderes, de un lado, y los afectados, sobre los cuales incidía la acción organizativa de los primeros" (1996, p. 137, traducción nuestra).

Ese movimiento se enfrentó a ELETROSUL, hizo paralizar la construcción de la represa de Itá en 1985, y en octubre de 1987 firmó un Acuerdo con la empresa, tanto para la represa de Itá como la de Machadinho, donde eran tomados en cuenta los propietarios como los no propietarios. Fue la primera vez en la historia de las luchas por la construcción de represas hidroeléctricas en Brasil, que los pequeños campesinos conseguían este triunfo. En el mismo se indicaban tres alternativas posibles para las unidades familiares rurales afectadas, como solución por el desplazamiento de las tierras ocupadas:

\footnotetext{
${ }^{6}$ Las irregularidades fueron enumeradas de la siguiente forma: “1 - ELETROSUL quien ya había negociado con 4 comunidades, todavía no había resuelto casos pendientes de comunidades anteriores; 2 - ELETROSUL no supo informar el precio de las tierras y mejoras. Ni había hecho reajuste sobre las investigaciones de precios realizadas en diciembre de 1987.3 - ELETROSUL no tenía tierras para reasentar a los afectados que tomaran la opción por el reasentamiento (...) . En otros municipios, había otros problemas: - Tala indiscriminada de árboles. - daños a vallas y cultivos. -Quema en las tierras de los afectados. - Entrada en las propiedades sin pedir permiso, ya estaban tornándose común donde había trabajo de demarcación”. (CRAB, 1988:1, traducción nuestra).
}

${ }^{7}$ Sobre el carácter de esta participación, consultar Scherer-Warren (1993).

DOI: https://doi.org/10.46699/rduno.v3i4.5879 | Edição Vol. 3, Núm. 4, 2020. 
1-La indemnización de tierras y mejoras.

2-El cambio de tierra por tierra.

3-El reasentamiento colectivo.

Diferenciamos, por un lado, este momento que fue un tiempo de victimización y de lucha política, que transformó al afectado en un sujeto social dispuesto a conquistar y garantizar derechos. Y por otro, un segundo momento, que lo denominamos tiempo de reconstrucción de los espacios físico y social donde los "atingidos" pasaron a ser "reasentados" lo cual presentó la posibilidad de acceso a la tierra como propietarios.

También hemos analizado cómo los "mediadores", en especial, la Iglesia Católica; la Iglesia Protestante de Confesión Luterana y los sindicatos rurales apoyaron y marcaron un antes y un después en la evolución de la CRAB.

Por último, queremos hacer nuestras las aseveraciones de Winckler y Renk que afirman que los proyectos hidroeléctricos, en el caso de Brasil, se apoyan en el discurso gubernamental de la necesidad de proveer infraestructura para el desarrollo, colocando en último puesto los deseos de la población afectada. Las autoras explican que los daños van más allá de la materialidad del patrimonio e incluyen la inmaterialidad de bienes y valores como la red de sociabilidad, la vecindad, la comunidad. (2019, p.90).

\section{BIBLIOGRAFÍA}

CATULlO, M. R. CIUDADES RELOCALIZADAS. Una mirada desde la Antropología Social. Buenos Aires: Editorial Biblos, 2006.

GERMANI, G. Expropriados. Terra e Agua: O conflito de Itaipú. Salvador: EDUFBA ULBRA, 2003 .

MAGALHÃES, S. O desencantamento da beira -reflexões sobre a transferência compulsória provocada pela usina hidrelétrica de Tucurui, In: MAGALHÃES, S. et al. (Org.) Energia na Amazônia. Museo Paraense Emílio Goeldi, Belém, Universidad Federal de Pará/Asociación de Univ. Amazónicas, 1996. p. 697-746.

MARTINS COSTA, A. L. B. Uma retirada insólita. A representação camponesa sobre a formação do lago de Sobradinho. Tesis de Maestria, Programa de Post-grado en Antropología Social, Museo Nacional, Rio de Janeiro, Universidad Federal de Rio de Janeiro, 1989.

MARTINS COSTA, A. L. B. Barragem de Sobradinho: O desencontro cultural entre camponeses é técnicos do Estado, In: A. L. B. MARTINS COSTA et al. Hidrelétricas, Ecologia e Progresso. Contribuções para um debate. Rio de Janeiro: CEDI, 1990. p. 55-68. 
MORAES, M.S.M. No rastro das águas, pedagogia do movimento dos atingidos pelas barragens da bacia do rio Uruguai (RS/SC) -1978/1990. Tesis de Doctorado en Educación, Rio de Janeiro, Pontificia Universidad Cátolica, 1994.

MORAES, M. S. M. No rastro das águas: organização, Liderança e representatividade dos atingidos por barragens. In: NAVARRO, Z. (Org.). Política, protesto e cidadania no campo: as lutas sociais dos colonos e trabalhadores rurais no Rio Grande do Sul. Porto Alegre: Editora da Universidade/UFRGS, 1996. p.137-169.

PANDOLFI, M.L. Na margem do lago. Um estudo sobre sindicalismo rural. Recife: Fundação Joaquim Nabuco/Editora Massangana, 1990.

REIS, M. J. Espaços vividos, migração compulsória, identidade. Os camponeses do Alto Uruguai e a hidrelétrica de Itá. Tesis de Doctorado en Ciencias Sociales. Programa de Post-grado en Ciencias Sociales, Campinas, Universidad Estadual de Campinas (UNICAMP), 1998.

REIS, M. J. Espaços, vivências e identidade: os camponeses do Alto-Uruguai e a Hidrelétrica de Ita, In: A. BALAZOTE, M. R. CATULLO y J. C. RADOVICH (Orgs.) Antropología y Grandes Proyectos en el Mercosur. La Plata: Editorial Minerva, 2001. p.89 -106.

RENK, A. y S. WINCKLER. De atingidos a vítimas do desenvolvimento: um estudo junto à população afetada direta ou indiretamente pela UHE Foz do Chapecó na região Oeste de Santa Catarina. In: Revista Direito Ambiental e Sociedade, v.7, n.2, 2017. p. 187-211.

ROCHA H.J da y G. de LIMA OLIVEIRA (2018) Resgate histórico-analítico da mobilização e organização dos atingidos por barragens na bacia do rio Uruguai (1970-2015), em J.C. Tedesco, J.J. Seminotti y H.J. da Rocha (orgs.) Movimentos e lutas sociais pela terra no sul do Brasil. Questões conteporâneas (On line). Chapecó, Editora UFFS. http://doi.org./10.7476/9788564905764.

ROTHMAN, F. D. A emergência do movimento dos atingidos pelas barragens da bacia do rio Uruguai (1979-1983). In: NAVARRO, Z. (Org.). Política, protesto e cidadania no campo: as lutas sociais dos colonos e trabalhadores rurais no Rio Grande do Sul. Porto Alegre: Editora da Universidade/UFRGS. 1996. p.106-136.

SCHERER-WARREN, I. Redes de Movimentos sociais. San Pablo: Loyola, 1993.

SCHERER-WARREN, I.; REIS, M. J. As barragems do Uruguai: a dinâmica de un movimiento social. Boletim de Ciências Sociais, Florianópolis, n. 41, 1986.

SCHERER-WARREN, I. y REIS, M.J. O Movimento dos Atingidos pelas Barragens do Uruguai: Unidade e Diversidade. In: Cadernos do CEAS, n. 120, Salvador, 1989.

SIGAUD, L. Efeitos Sociais de Grandes Proyetos Hidrelétricos: As barragens de Sobradinho e Machadinho, Comunicación No 9, Programa de Post Grado en Antropologia Social, Museo Nacional Rio de Janeiro, UFRJ, 1986. 
SIGAUD, L. Implicações sociais da política do setor elétrico. In: SANTOS, L. y ANDRADE L. As Hidrelétricas do Xingú e os povos indígenas. San Pablo: Comissão Pró-Indio, 1988, p. 103110 .

VAINER, C. B y ARAÜJO, F.C.B. Implantações de grandes hidrelétricas: estratégias do Sector elétrico, estratégias das populações atingidas. Revista Travessia, no 6, enero-abril, 1990. p. 18-24.

WINCKLER, S., y RENK, A. (2019). Víctimas del desarrollo de empresas hidroeléctricas en el sur de Brasil. Administración Pública Y Sociedad (APyS), (8), 82-93. Recuperado a partir de https://revistas.unc.edu.ar/index.php/APyS/article/view/26390. Acceso: 17 de abril de 2020.

\section{DOCUMENTOS}

CRAB, Periódico “A Enchente do Uruguai”, n 2.1982

CRAB, Periódico “A Enchente do Uruguai” n11. 1985

CRAB, Periódico “A Enchente do Uruguai”, n 15.1986

CRAB, Nova história em debate, Erexim, Gráfica Vianei.1990

ELETROSUL/CNEC "Relatório de Impacto Ambiental Usina Hidrelétrica de Itá", Florianópolis. 1990 